\title{
Protein intake by gypsy moth larvae on homogeneous and heterogeneous diets
}

\author{
BRIAN A. STOCKHOFF Department of Biology, University of Michigan, Ann Arbor, \\ Michigan, U.S.A.
}

\begin{abstract}
Food selection behaviour, food utilization efficiency and growth performance of a generalist insect, the gypsy moth (Lymantria dispar (L.), Lepidoptera: Lymantriidae), were examined with respect to variation in food nitrogen concentration. The results suggest that gypsy moth do not suffer physiologically and in fact may benefit from intraplant variation by selective feeding. When provided with diet cubes containing identical nitrogen concentrations, control larvae tended to consume food from a single cube. This behaviour contrasted with that of larvae provided cubes differing in nitrogen concentration. These larvae tended to consume more from the high nitrogen cube, but allocated feeding more evenly among diet cubes than did control larvae. Overall, larvae mixed foods so as to obtain a mean concentration of $2.9-3.2 \%$ nitrogen, a concentration assumed to approximate the 'intake target'. Larvae confined to single nitrogen concentrations mitigated the impact of imbalanced diets on body composition via both pre-ingestive and post-ingestive compensation. When confined to a specific nitrogen concentration, larvae adjusted their intake to the point of best compromise. In this case, this was the geometrically closest point to the estimated intake target. Larvae with a choice of foods that deviated more than $\pm 1 \%$ from each other in nitrogen concentration grew as well as or better than larvae without a choice but given identical mean nitrogen concentrations. These results demonstrate that selectivity and nitrogen consumption by gypsy moth larvae are altered according to the particular choices available. Insects may benefit from intraplant variation in food quality because such variation provides the opportunity to choose foods and mix them in ways that permit close matching with the intake target. Variation may be particularly important to insects which must offset changing nutritional demands.
\end{abstract}

Key words. Compensatory feeding, gypsy moth, nutrition, nitrogen, diet heterogeneity, variability, foraging behaviour, Lymantria dispar.

\section{Introduction}

Variability in food nutritional quality is encountered by all phytophagous insects (Scriber \& Slansky, 1981; Slansky \& Scriber, 1985). If an insect is unable to make choices among available items, variation in food quality has the potential to suppress growth because a large proportion of foods encountered and consumed may deviate significantly from the mean or ideal food type (Stockhoff, 1993a). On the other hand, if an insect can select, variation might

Correspondence: Dr B. A. Stockhoff, Mycogen Corporation, 4980 Carroll Canyon Road, San Diego, CA 92121, U.S.A. enhance growth if an insect were to choose a mixture of foods that more closely matches its nutritional requirements than could a single food item.

Demonstration that an insect actively regulates its nutritional intake by selective feeding requires evidence that the balance and quantity of ingested nutrients meet current demands better than by eating the same sources of nutrients randomly or in any other combination. Insects feeding in such a manner have been termed 'self-selectors' (Waldbauer \& Friedman, 1991). Many studies demonstrate non-random food selection, but only a few examples exist where the selected ratio of foods is shown to be superior to other possible ratios. Recently, such studies with insects 
have involved presentation of two artificial diet cubes or cakes (Waldbauer et al., 1984; Cohen et al., 1987a; Schiff et al., 1988; Bernays \& Bright, 1991). In control treatments, the cubes are identical and nutritionally complete. In experimental treatments, the cubes are complementary in that each cube is deficient in a different nutrient, but otherwise is nutritionally complete. Control insects tend to restrict feeding to a single cube, whereas experimental insects divide feeding more equally among the cubes. For example, nymphs of the brown-banded cockroach, Supella longipalpa (Cohen et al., 1987a), and corn earworm larvae, Helicoverpa zea (Waldbauer et al., 1984), obtained a favourable balance of protein and carbohydrate by nonrandom feeding from complementary diet cubes, each deficient in one of these nutrients but otherwise complete. The capacity for selection is not limited to macronutrients in $H . z e a$, which can also self-select required vitamins and lipid (Schiff et al., 1988). Combined with analyses of growth and food utilization, these observations clearly indicate a capacity to alter feeding in response to available foods, and that mixing of deficient but complementary foods is beneficial.

Complementarity of food items arises when items covary negatively in nutritional qualities. In an extreme case, essential nutrients may be acquired only by consuming different food types (Tilman, 1980). By definition, then, fitness may be increased only through mixing of food types. However, natural foods such as leaves are characterized by continuous variation in nutrients that often positively, not negatively, covary (Scriber, 1984). Thus, among the leaves selected by a typical insect herbivore, strong complementarity between individual leaves will be rare. The majority will be substitutable in that they are qualitatively similar and nutritionally equivalent at some exchange rate. It has not previously been shown that any insect can self-select when provided with strongly substitutable rather than complementary foods. A fundamental difference in behaviour might be expected in response to complementary and substitutable foods. Growth on the former requires mixing of foods, whereas growth on the latter may be optimal on food items described by a narrow range within the continuum. In either case, mixing might provide an optimum which does not exist in nature or is rare. Because no published studies have examined self-selective behaviours in response to substitutable foods with continuous variation, whether insects respond differently to substitutable and complementary foods is unknown.

It is useful to consider food selection in terms of the 'nutritional target' and 'intake target' (Raubenheimer \& Simpson, 1993). The nutritional target for protein is that amount of protein which will provision tissues at the optimal level. The intake target is the amount of protein that is needed to be consumed so that post-ingestive utilization permits the nutritional target to be reached with maximal efficiency. Larvae confined to a single food will consume nutrients in fixed proportions, or 'rails' (Raubenheimer \& Simpson, 1993), and can match their intake target only if the intake target lies on the rail.
Compromise is necessary if nutrients are not in the optimal balance. These larvae can meet their nutrient targets by altering consumption to the point of best compromise and then 'jumping rails' via post-ingestive processes, selectively utilizing ingested nutrients. Precisely what defines this point of compromise will depend on ecological and physiological constraints, as well as the evolutionary history of the insect. On the other hand, larvae with a choice of foods have the opportunity to match more closely their intake target, and may need to rely less heavily on post-ingestive processes in order to reach their nutritional target.

In this study I examine the outcome of food selection behaviour and the impact of different choices on food utilization efficiency and growth performance of a generalist insect, the gypsy moth (Lymantria dispar (L.)) with respect to food nitrogen concentration. Nitrogen is extremely important to growth of juvenile insects, and is known to vary substantially within and among individual host plants (Slansky \& Scriber, 1985; Gulmon \& Chu, 1981; Hollinger, 1989). As a result, choices are available to foraging larvae. The central aim was to determine if larvae with a choice of foods gain an advantage over larvae that do not have a choice. The experiments addressed three primary questions: (1) Do gypsy moth larvae distinguish foods differing in nitrogen concentration? (2) Do larvae have a functional feeding rule such that individuals confined to single protein concentrations reach predictable points of best compromise? (3) Do larvae provided with different protein concentrations have a well-defined protein intake target?

\section{Materials and Methods}

Diet treatments. Foliage can be quite variable such that a wide array of nitrogen concentrations may be available to a foraging insect at a single point in time. Nitrogen concentrations in tree leaves typically range from 0.6 to $6.9 \% \mathrm{dw}$ with a mean around $2.5-3.0 \%$ (Slansky \& Scriber, 1985). Variation can be great even within individual plants; leaves within single ramets of Populus tremuloides, for example, have been found to range in nitrogen concentration from $1.2 \%$ to greater than $4.5 \%$ (Stockhoff, 1992). Hence, diet treatments were created to test the response of gypsy moth larvae to foods across this general range of nitrogen, with a mean of $3.0 \%$.

Five artificial diets were prepared based approximately on the Bell diet for gypsy moth (Odell et al., 1985). Common to each diet were the following ingredients (for 1 litre of finished diet): wheat germ $(30.6 \mathrm{~g})$, BioServ Lepidopteran salt mix $(7.8 \mathrm{~g})$, choline chloride $(0.6 \mathrm{~g})$, ascorbic acid $(5.5 \mathrm{~g}), p$-methylhydroxybenzoic acid $(1.2 \mathrm{~g})$, sorbic acid $(2.2 \mathrm{~g})$, dextrose $(3.3 \mathrm{~g})$, soluble potato starch $(20 \mathrm{~g})$, linseed oil $(8.0 \mathrm{~g})$, BioServ Lepidopteran vitamin mix $(0.8 \mathrm{~g})$, agar $(25 \mathrm{~g})$, and distilled, deionized water $(805 \mathrm{ml})$. Diets differed in vitamin-free casein concentration. Diets containing $1.25,2.25,3.00,3.75$ and $4.75 \%$ nitrogen $(\mathrm{N})$ contained $0,15,25,35$ and $50 \mathrm{~g}$ casein $/ \mathrm{l}$, respectively. 
Nitrogen not provided by casein originated from wheat germ. To prevent dilution of other components as nitrogen was increased, non-nutritive cellulose was added in corresponding amounts of $92,77,67,57$ and $42 \mathrm{~g} / \mathrm{l}$. These diets were substitutable with regard to nitrogen in order to focus on response of larvae to choice of nitrogen concentrations. Diets were partially rather than perfectly substitutable in that not all nutrients were altered identically in a dilution series.

Fifteen diet combinations were established as treatments (Table 1). These were divided into Low-Variance and High-Variance categories which, pooling across diet treatments, centred around a mean nitrogen concentration of $3.0 \%$. Low-Variance treatments comprised various combinations of $2.25,3.00$ and $3.75 \%$ nitrogen; HighVariance treatments comprised combinations of $1.25,3.00$ and $4.75 \% \mathrm{~N}$. Low-Variance treatments were set arbitrarily to have a smaller range in $\% \mathrm{~N}$ than High-Variance treatments, but with identical mean $\% \mathrm{~N}$. In each Variance category, three treatments involved provision of larvae with two diet cubes of the same nitrogen concentration (treatments $1-3$ for High-Variance, treatments 8-10 for Low-Variance). In remaining treatments (treatments 4-7, $11-15)$, larvae were provided with two, three or five diet cubes of differing nitrogen concentration. Diet treatments in which two cubes of the same nitrogen concentration were provided $(1-3,8-11)$ collectively were classified as No Choice treatments, and remaining treatments (4-7, $11-15)$ were designated as Choice treatments. Because all larvae received at least two cubes, these designations refer to choice between different cubes (Choice) as opposed to identical cubes (No Choice).

Seven of the diet treatments $(2,5$ and 7 in the HighVariance category and 9,12, 14 and 15 in the Low-Variance category) had identical mean nitrogen concentration of $3.0 \%$, but different variances around the mean. Col-

Table 1. Nitrogen concentration of diet cubes provided to larvae and the mean nitrogen concentration of these cubes.

\begin{tabular}{llll}
\hline $\begin{array}{l}\text { Variance } \\
\text { treatment }\end{array}$ & $\begin{array}{l}\text { Diet } \\
\text { treatment }\end{array}$ & $\begin{array}{l}\text { \% N of cubes } \\
\text { provided }\end{array}$ & $\begin{array}{l}\text { Mean \% N } \\
\text { provided }\end{array}$ \\
\hline High Variance & 1 & 1.25 & 1.25 \\
& 2 & 3.00 & 3.00 \\
& 3 & 4.75 & 4.75 \\
& 4 & $1.25,3.00$ & 2.12 \\
& 5 & $1.25,4.75$ & 3.00 \\
Low Variance & 6 & $3.00,4.75$ & 3.88 \\
& 7 & $1.25,3.00,4.75$ & 3.00 \\
& 9 & 2.25 & 2.25 \\
& 10 & 3.00 & 3.00 \\
& 11 & 3.75 & 3.75 \\
& 12 & $2.25,3.00$ & 2.62 \\
& 13 & $2.25,3.75$ & 3.00 \\
& 14 & $3.00,3.75$ & 3.38 \\
& 15 & $2.25,3.00,3.75$ & 3.00 \\
& & $1.25,2.25,3.00$, & 3.00 \\
\hline
\end{tabular}

lectively, variances were higher for the High-Variance diet treatments than the Low-Variance diet treatments. Note that treatments 2 and 9 are replicates of the $3.0 \% \mathrm{~N}$ No Choice treatment. This was done so that each variance category would have its own, separate $3.0 \% \mathrm{~N}$ group for use in statistical tests. Also, although treatment 15 (T15) included 1.25 and $4.7 \% \mathrm{~N}$ diets used in the High-Variance experiment, it was included with the Low-Variance treatments because the treatment was carried out concurrent with the other Low-Variance treatments.

Larvae. Egg masses were collected from a newly-infested forest in Roscommon Co., Michigan, in late winter 1990. Three egg masses each were assigned to Low-Variance and High-Variance categories. Egg masses were kept at $5^{\circ} \mathrm{C}$ until transfer to an incubator for hatching in mid-May. Egg masses were split lengthwise with one half assigned to the Low-Variance treatment, the other assigned to the High-Variance treatment. Hence, the eggs were assumed to be randomly divided between the Low- and HighVariance treatments. Lengthwise division ensured that each treatment group received both first- and last-laid eggs from the same mass. Half-masses assigned to the High-Variance treatments were hatched 9 days prior to those in the Low-Variance treatments in order to distribute the required work over time. The drawback to this design is that results from High- and Low-Variance treatments are not strictly comparable, due to potential effects associated with time.

Following hatching, thirty neonates from each egg mass were assigned randomly to each of the fifteen diet treatments, yielding a total of ninety larvae per diet treatment. The thirty larvae provided measures for food choice (eighteen larvae) and fresh to dry mass conversion (twelve larvae). Ten of the eighteen larvae were used in calculation of nutritional indices. Larvae were reared on assigned treatments for the entire juvenile period, beginning 1 day after hatching. Larvae were maintained in $30-\mathrm{ml}$ plastic cups with lids; those exceeding $1 \mathrm{~g}$ fresh weight (primarily females entering the sixth instar; males typically have five instars only) were transferred to $100 \times 15 \mathrm{~mm}$ plastic petri dishes and maintained in these until pupation. Hatching and rearing were in incubators at $25^{\circ} \mathrm{C}$ and $\mathrm{LD} 16: 8 \mathrm{~h}$.

Food choice. Food choice by larvae in each diet treatment was evaluated during the fourth stadium. Upon entry to the fourth stadium, larvae were weighed and provided fresh diet cubes of known mass. Cubes provided to individuals were approximately identical in size, and food generally was available in sufficient quantity to last the stadium. Cubes were arbitrarily designated A and B in treatments in which cubes were identical in nitrogen concentration. An aliquot of each diet was taken each day to determine percentage dry mass for fresh to dry mass conversion. To avoid bias caused either by relative diet position or treatment location within the incubators, diets were placed in a random arrangement within rearing cups, and trays of cups were shifted daily in their position within the incubators.

Food choice trials were terminated under any of three conditions: (1) larvae initiated moulting to the fifth stadium, 
(2) $>80 \%$ of any diet cube was consumed, or (3) after 3 days, whichever came first. These conditions minimized disturbance to larvae (which might affect food choice), reduced potential effects of changes in the relative abundance of diet types on preference, and ensured that assays were made over a similar period of time. Upon termination of a trial, uneaten food was removed, dried to constant mass at $70^{\circ} \mathrm{C}$, and weighed.

Food consumption and utilization. Upon entry to the fourth stadium, approximately thirty larvae from each diet treatment were weighed, and freshly-weighed diet cubes were placed into rearing cups, as in the food choice trials. Mean larval mass was statistically identical among treatment groups (data not shown). Diet was resupplied if the stadium lasted longer than 3 days. After cessation of feeding before moulting into the fifth stadium, larvae were reweighed, and frass and uneaten diet were dried at $70^{\circ} \mathrm{C}$ and weighed. Two measures of consumption, relative consumption rate (RCR) and relative nitrogen consumption rate (RNCR), and standard nutritional indices were calculated on a dry mass basis (Waldbauer, 1968). Indices were:

$$
\begin{aligned}
& \text { RCR: Relative consumption rate } \\
& =\frac{\text { food ingested }}{\text { mean larval mass } \times \text { days }}
\end{aligned}
$$

RNCR: Relative N consumption rate

$$
=\frac{\text { nitrogen ingested }}{\text { mean larval mass } \times \text { days }}
$$

AD: Assimilation efficiency

$$
=\frac{\text { food ingested }- \text { frass }}{\text { food ingested }} \times 100
$$

ECI: Net growth efficiency

$$
=\frac{\text { biomass gained }}{\text { food ingested }} \times 100
$$

ECD: Gross growth efficiency

$$
=\frac{\text { biomass gained }}{\text { food ingested }- \text { frass }} \times 100
$$

So as to remove the purely physical influence of indigestible cellulose, AD, ECI and ECD were calculated for nutrients only by subtracting cellulose from ingestion and egestion figures. The amount of nitrogen ingested was determined by mutiplying the amount eaten from each diet cube by the nitrogen concentration of that diet and summing the resulting values. Percentage nitrogen eaten in aggregate over the stadium was calculated by dividing the total mass of nitrogen consumed by the total mass of food consumed $(\times 100 \%)$. Mean larval mass during the stadium was calculated using the arithmetic mean of initial and final mass upon entry to and exit from the fourth stadium.

Note that in food choice experiments such as this it is virtually impossible to obtain deal conditions for analysis of food utilization (van Loon, 1991). In particular, to minimize error in calculation of utilization indices, Schmidt \& Reese (1986) suggested provisioning larvae so that greater than $80 \%$ of the food provided to larvae is consumed. Clearly this is not possible if larvae preferentially consume one diet cube, as occurred in No Choice treatments. Hence, error in calculated indices likely is greater in the No Choice treatments. However, the potential for bias was reduced in No Choice treatments by adjusting the initial amounts provided so that the excess food remaining was minimized and roughly equal across treatments at the end of a trial. Specifically, larger cubes were provided as nitrogen concentration decreased. In Choice treatments, the total amount of excess food was reduced successfully because larvae typically ate substantial amounts from all available cubes.

Pupal mass, development time and growth rate. To determine the effects of prolonged diet variation on fitness-related characters, juvenile growth parameters were measured for larvae used in food choice trials. Development time was defined as the period between hatch and the formation of a hardened pupal case. Mass of pupae was determined at this time, and development was allowed to continue to eclosion for sex determination. Growth rate was determined as pupal dry mass/development time.

Data analysis. Because no single measure of consumption accurately reflects choice behaviour, food choice data were analysed by two statistics. First, Chesson's alpha (Chesson, 1983; Lechowicz, 1982) was calculated as an index of food choice using the form

$$
\text { alpha }=\frac{r_{i} / n_{i}}{\sum_{j=1}^{m} r_{j} / n_{j}}, i=1, \ldots, m
$$

where $r_{i}$ and $n_{i}$ are the percentages of food type $i$ in the diet and environment, respectively, and $m$ is the number of food types available. Chesson's alpha was calculated for each diet type provided and compared with an expected value of $1 / m$ where $m$ is the number of cubes provided. An alpha significantly different from expected indicates the food type was consumed out of proportion to its relative abundance. Because the masses of diet cubes provided to individuals were roughly the same, Chesson's alpha is approximately equivalent to the percentage that each diet cube contributed to the total eaten.

A second measure of food choice was a Levene's test for equality of variance (van Valen, 1978, as modified by Waldbauer \& Friedman, 1988). The percentage that each diet cube contributed to the total food eaten was calculated, and the average difference between these was found. This modified Levene's number was compared between Choice and No Choice treatments. A large modified Levene's number indicates that individuals consumed the diet cubes in very different amounts, whereas a small modified Levene's number indicates that feeding by individual larvae was divided evenly among the diet cubes. Note that, unlike Chesson's alpha, the modified Levene's number is 
sensitive only to the difference in amount consumed between the cubes and is insensitive as to which cube is consumed. For example, if $80 \%$ of the total amount eaten came from one of two cubes, the modified Levene's number would be the same $(0.8-0.2=0.6)$ regardless of which cube contributed the $80 \%$. The statistic can be calculated for any pair of diets, but analysis was restricted to diet treatments in which a maximum of two cubes were provided because differences among multiple pairings are difficult to interpret.

Juvenile growth parameters which satisfied assumptions of homogeneity of variances and normality were analysed by parametric tests. For the High Variance treatments this included the following variables: male pupal mass and growth rate, and development time of both males and females. Heteroscedasticity of data prevented parametric analysis of certain variables, including female pupal mass and growth rate. These variables were analysed nonparametrically. Accordingly, $t$-tests or Mann-Whitney U-tests were performed among selected pairs of treatments to compare (1) growth on Choice treatments with that achieved on the component diets (e.g. T6 $(3.0 \%, 4.75 \% \mathrm{~N})$ versus $\mathrm{T} 2(3.0 \% \mathrm{~N})$ and T3 $(4.75 \% \mathrm{~N}))$, and (2) growth on Choice treatments with mean nitrogen concentration provided of $3.0 \%$ with that on $3.0 \% \mathrm{~N}$ No Choice treatments (e.g. T11 $(2.25 \%, 3.75 \% \mathrm{~N})$ versus T9 $(3.0 \% \mathrm{~N}))$. Although all possible pairwise comparisons were not performed, many treatments entered into statistical tests more than once. To provide a conservative correction for the number of comparisons, an $\alpha$ adjusted as $0.05 / n$ should be applied to determine significance of $P$-values, where $n$ is the number of comparisons.

Consumption rates and nutritional indices were compared between Choice and No Choice treatments by ANOVA. In order to compare overall responses by larvae with versus without a choice of protein, values from treatments with $3.0 \% \mathrm{~N}$ provided were pooled and then compared between Choice and No Choice categories. Prior to analysis, RNCR was reciprocal-transformed to equalize variances. Plots of protein consumption were made in order to estimate the intake and nutritional targets, and to determine how larvae adjusted their feeding relative to the intake target.

\section{Results}

Food choice

Provided with a choice of two diet cubes, larvae given identical cubes (treatments $1-3,8-10$ ) consumed $50 \%$ of their total food intake from each cube when averaged across all individuals (Chesson's alpha for cube A compared with $50 \%$ for High-Variance treatments T1-T3: alpha $=0.50$, df $=121, t=0.13$, NS; Low-Variance treatments T8-T10: alpha $=0.52, \mathrm{df}=105, t=0.87, \mathrm{NS}$ ). In contrast, in the High-Variance experiment larvae provided with two cubes that differed in nitrogen concentration ate less overall from the cube with lower nitrogen (Chesson's alpha $=0.44, \quad \mathrm{df}=114, \quad t=3.18, \quad P<0.01$;
Fig. 1). Larvae in the Low-Variance treatments ate equal amounts from each cube (Chesson's alpha $=0.54, \mathrm{df}=135$, $t=1.93$, NS).

Although larvae as a group ate approximately $50 \%$ from each cube when cubes were identical, individual larvae did not divide their feeding equally between the two cubes. Larvae given identical cubes in the HighVariance experiment had a higher mean difference between the percentage of the total food eaten contributed by each cube than did larvae provided different cubes (modif:ed Levene's number for No Choice (42.2) versus Choice (35.1): $t=2.00$, df $=235, P<0.05$ ). This indicates that larvae provided with cubes of different $\mathrm{N}$ concentrations tended to divide their feeding more equally between the cubes than did larvae with only a single nitrogen concentration. Larvae in the Low-Variance experiment did not respond as strongly and divided their feeding among identical cubes much as they divided feeding among different cubes (modified Levene's for No Choice $=34.1$, Choice $=35.1 ; \mathrm{df}=240, t=0.30, \mathrm{NS}$ ).

Because the feeding by Low-Variance larvae could not be distinguished conclusively from random feeding based on either Chesson's alpha or modified Levene's tests, detailed statistical comparisons will be shown only for larvae experiencing the High-Variance treatments, although intake measures from all treatments will be presented in figures. It is important to note that the inability to distinguish feeding from random does not necessarily imply that larvae were not regulating nutrient intake (see Intake and nutritional targets, below). The limited response by Low-Variance larvae was not unexpected because the cubes presented were more similar in the Low-Variance experiment than in the High-Variance experiment.

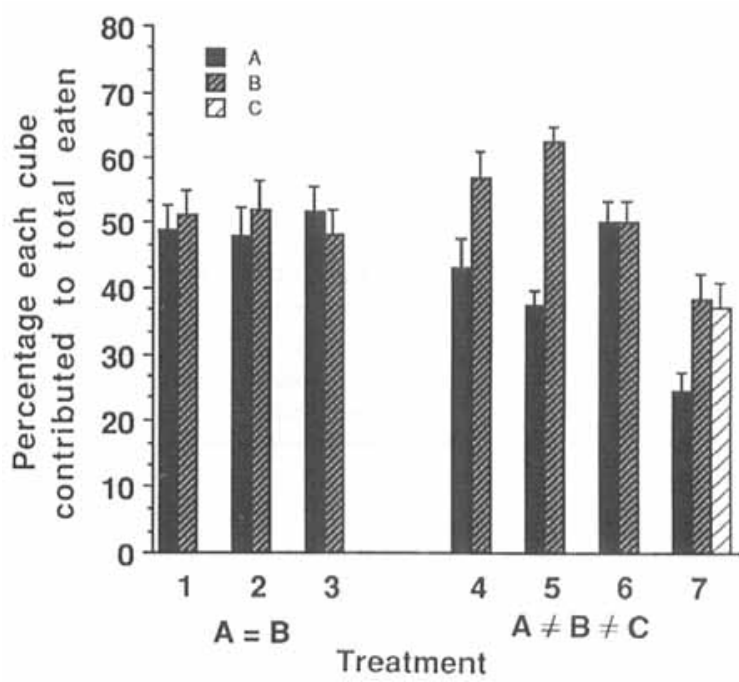

Fig. 1. Mean percentage each diet cube $(A, B, C)$ contributed to the total amount eaten. In treatments 4-7, diet cube A contained the least nitrogen. Diet cube $\mathrm{C}$ contained the most nitrogen (in treatment 7 only). Error bars represent \pm 1 SEM. Sample sizes for treatments $1-7$ were $39,37,46,26,44,45$ and 41 , respectively. 
Larval growth

As indicated above, larvae in the High-Variance experiment tended to mix different diet cubes more than they mixed identical diet cubes. If there is a growth advantage to this behaviour, then growth should be: (1) greater in Choice treatments than in the No Choice treatments in which individual component diet $\mathbf{N}$ concentrations were provided, and (2) greater in Choice treatments than in No Choice treatments in cases in which larvae were provided with the same mean $\mathrm{N}$ concentration. These will be discussed in turn.

Growth on Choice diets versus No Choice components. For both male and female larvae, growth performance in treatments where choice was possible was greater than or equal to that achieved on the component diets (Table 2). Differences in pupal mass were statistically significant in all comparisons with T1 (males: all $t>3.7, P<0.001$; females: all $U<0.001, P<0.001)$. Development tended to be more rapid in Choice than No Choice treatments, with the effect being stronger for males. Overall, larvae with greater flexibility in protein consumption had faster growth rates (Fig. 2).

An important subset of the Choice versus No Choice comparisons is that in which the high $\mathrm{N}$ cube of the Choice treatment was equal to the percentage $\mathrm{N}$ in the No Choice treatment (T4 v T2, T5 $v$ T3, T6 v T3, T7 v T3). In these situations, it was not possible for larvae in the Choice treatments to exceed the nitrogen intake in the No Choice treatments. In these comparisons, male larvae in the Choice treatments consistently had marginally improved performance, with higher pupal mass (T7 $v$ T3: $t=2.58$, $\mathrm{df}=18, P=0.019$ ), more rapid development (T4 $v \mathrm{~T} 2$ : $t=1.88, \mathrm{df}=15, P=0.079 ;$ T5 $v$ T3: $t=2.10, \mathrm{df}=21$, $P=0.048$ ), and higher growth rates (T4 $v \mathrm{~T} 2: t=2.88$, $\mathrm{df}=15, P=0.011 ; \mathrm{T} 7 v \mathrm{~T} 3: t=2.03, \mathrm{df}=18, P=0.057)$. Responses by female larvae in these comparisons were statistically identical between the Choice and No Choice groups except for juvenile development time (T4 $v$ T2: $t=3.34$, df $=28, P=0.002$ ). Note that interpretations are

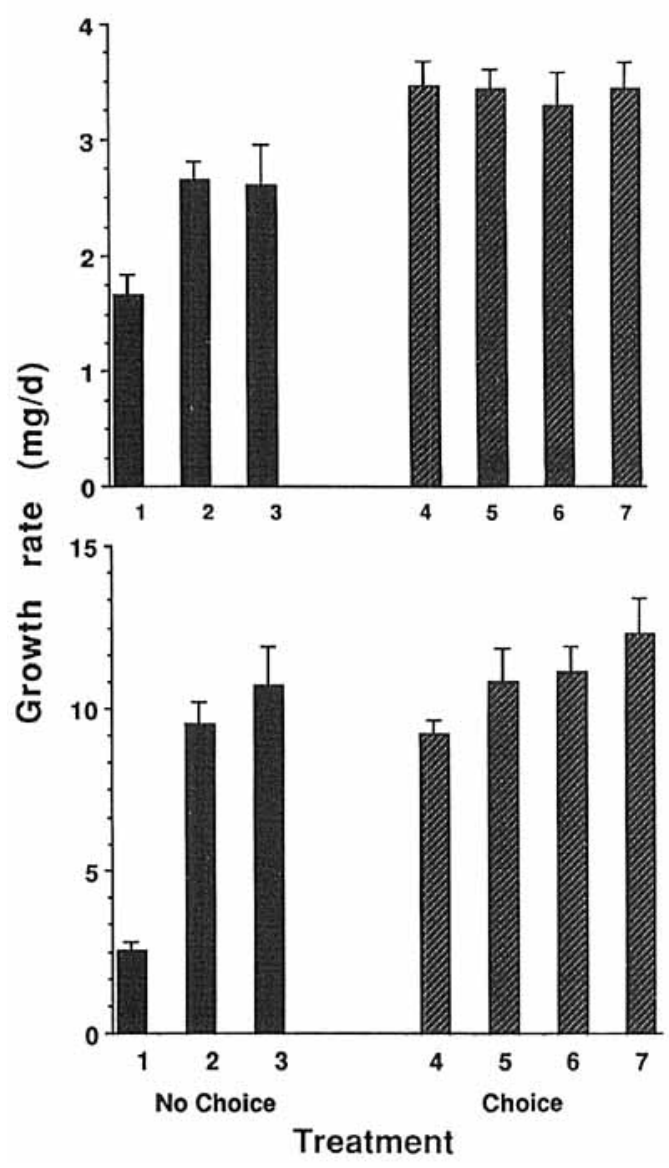

Fig. 2. Growth rate of male (upper panel) and female (lower panel) gypsy moth larvae reared on High-Variance diets differing in nitrogen concentration. Error bars represent \pm 1 SEM. Samplc sizes are listed in Table 2.

properly made after correcting for multiple comparisons $(\alpha=0.05 / 4=0.0125)$.

Growth on Choice versus No Choice treatments with identical mean $N$ concentration of $3.0 \%$. The results are

Table 2. Mean (SEM) pupal dry mass and development time of male and female gypsy moth larvae reared on High Variance diets differing in nitrogen concentration. Units are $\mathrm{mg}$ (d.w.) and days for pupal mass and juvenile period, respectively. NC indicates No Choice treatments (identical cubes provided) and $\mathrm{C}$ indicates Choice treatments (different cubes provided).

\begin{tabular}{|c|c|c|c|c|c|c|}
\hline \multirow[b]{2}{*}{ Treatment } & \multicolumn{3}{|c|}{ Male } & \multicolumn{3}{|c|}{ Female } \\
\hline & $n$ & $\begin{array}{l}\text { Pupal } \\
\text { mass }\end{array}$ & $\begin{array}{l}\text { Juvenile } \\
\text { period }\end{array}$ & $n$ & $\begin{array}{l}\text { Pupal } \\
\text { mass }\end{array}$ & $\begin{array}{l}\text { Juvenile } \\
\text { period }\end{array}$ \\
\hline $1(\mathrm{NC})$ & 14 & $65.0(5.9)$ & $41.7(3.0)$ & 8 & $134.7(9.0)$ & $55.0(5.1)$ \\
\hline $2(\mathrm{NC})$ & 8 & $83.3(8.3)$ & $31.1(1.5)$ & 14 & $320.4(21.0)$ & $33.8(0.6)$ \\
\hline $3(\mathrm{NC})$ & 9 & $75.1(8.2)$ & $30.4(1.7)$ & 14 & $352.4(34.3)$ & $33.6(0.8)$ \\
\hline $4(C)$ & 9 & $95.5(4.6)$ & $28.0(0.8)$ & 16 & $334.0(13.3)$ & $36.5(0.5)$ \\
\hline $5(C)$ & 14 & $93.0(4.6)$ & $27.3(0.5)$ & 11 & $367.8(27.0)$ & $35.1(1.5)$ \\
\hline $6(\mathrm{C})$ & 7 & $90.4(7.8)$ & $27.6(0.5)$ & 15 & $378.9(22.1)$ & $34.5(0.8)$ \\
\hline 7 (C) & 11 & $97.7(4.2)$ & $29.0(0.8)$ & 11 & $403.4(32.2)$ & $33.2(0.8)$ \\
\hline
\end{tabular}


particularly interesting when the No Choice treatments are compared with Choice treatments having the same mean $\% \mathrm{~N}$ as the No Choice treatments. Comparison was possible for treatment $\mathrm{T} 2$ versus (T5, T7), as each treatment provided $3.0 \% \mathrm{~N}$ to the larvae (Table 2, Fig. 2). Overall, larvae performed at least as well on Choice treatments having a mean of $3.0 \% \mathrm{~N}$ as on the No Choice treatment, also with $3.0 \% \mathrm{~N}$. Pupal mass was numerically greater in Choice treatments (T5, T7) than No Choice Treatment 2 , but not significantly so for males $(t=1.64$, df $=31, P=0.112)$ or females $(\mathrm{U}=93.0, n=14,22$, $P=0 .(48)$. Development time by males was significantly shorter in Choice treatments than No Choice $(t=2.59$, $\mathrm{df}=31, P=0.014)$, but not for females $(t=0.298, \mathrm{df}=34$, $P=0.768)$. Growth rate was greater for males $(t=2.95$, df $=31, P=0.006)$ but was not significantly so for females $(\mathrm{U}=101.0, n=14,22, P=0.085)$. For these comparisons, $\alpha$ should be set at $\alpha=0.05 / 2=0.025$, adjusting for the two comparisons. In brief, differences in growth were statistically marginal for many of the pairwise comparisons, but the overall trend was for larvae to benefit from having a choice of food nitrogen concentrations.

\section{Intake and nutritional targets}

Larvae in $\mathrm{T} 5$ and $\mathrm{T} 7$, in which larvae were provided with mean nitrogen concentrations of $3.0 \%$, chose the diet cubes such that the nitrogen concentration of the diet consumed exceeded $3.0 \%$ (T5: mean $=3.28 \%, t=3.87$, df $=44, P<0.001 ; \mathrm{T} 7:$ mean $=3.23 \%, t=2.90, \mathrm{df}=40$, $P<0.01)$. Despite this, larvae in the High Variance treatments with a choice of food $\mathrm{N}$ concentrations consumed food and nitrogen at lower rates than larvae without a choice. RCR declined in both Choice and No Choice treatments as the perentage $\mathrm{N}$ offered to larvae increased (Table 3). Given the same mean concentration of $\mathbf{N}$, however, larvae in Choice treatments had lower RCR than in No (hoice treatments $(t=6.1$, df $=87, P<0.001)$. Because larvae provided with a choice had the potential to consume a high nitrogen diet, it was possible for larvae in Choice treatments to consume food at a lower rate yet achieve the same nitrogen consumption rate as No Choice larvae. This was not the case; larvae given the same mean percentage nitrogen but with a choice had substantially lower RNCR than larvae in No Choice treatments $(t=4.9$, df $=87, P<0.001$; Table 3 ).

Larvae provided foods differing in protein concentration consumed, on average over all treatments, $31.6 \mathrm{mg}$ of protein and $144.3 \mathrm{mg}$ of non-protein food. This yielded a selected concentration of approximately $18 \%$ protein, or $2.9 \% \mathrm{~N}$ (Fig. 3). These values provide an estimate of the intake target which can be compared with intake by larvae confined to particular protein concentrations (Raubenheimer \& Simpson, 1993). Assuming the intake target does not lie precisely on the nutrient 'rail', larvae that are unable to choose must find the point of best com-

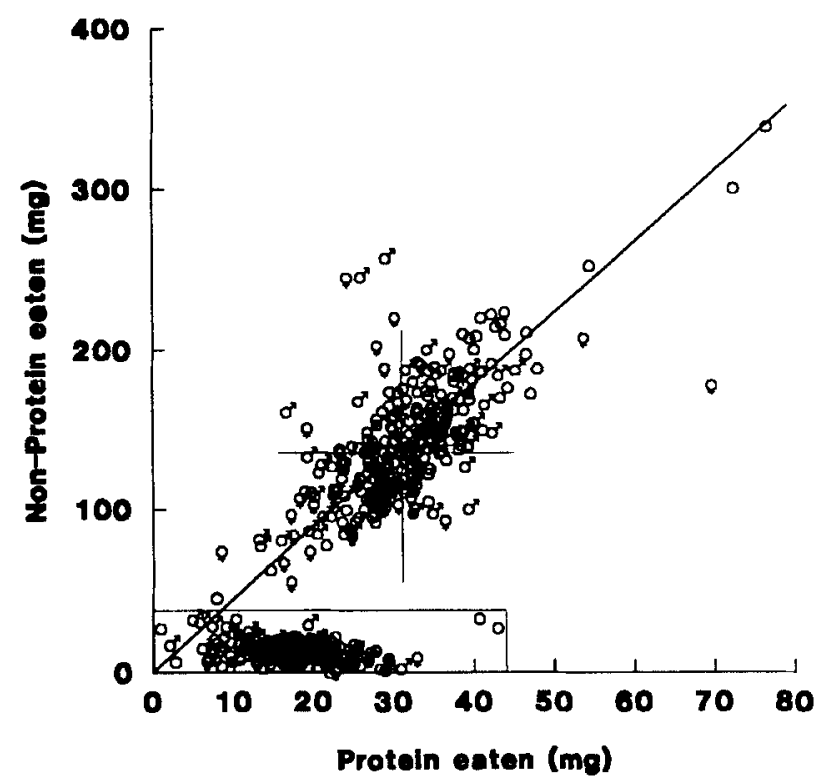

Fig. 3. Intake and growth targets by Choice larvae as defined by the amounts of protein and non-protein diet components ingested. The cross-hairs indicate the intake target, which lies along the line having a slope equal to the ratio of protein:non-protein eaten. The box near the origin surrounds the estimated growth target of larvae. Open circles designate larvae for which sex was not determined.

Table 3. Mean (SEM) consumption rates and food utilization efficiencies for fourth-stadium gypsy moth larvae in the High Variance treatment. Units are $\mathrm{mg} / \mathrm{mg} \mathrm{d}$ and $\mu \mathrm{g} / \mathrm{mg} \mathrm{d}$ for RCR and RNCR, respectively, and \% for $A D, E C D$ and ECI.

\begin{tabular}{llllllr}
\hline \multirow{2}{*}{$\begin{array}{l}\text { Treatment } \\
\text { (mean \%N) }\end{array}$} & $n$ & RCR & RNCR & AD & \multicolumn{1}{l}{ ECD } & \multicolumn{1}{l}{ ECI } \\
\cline { 2 - 7 } $1(1.25)$ & 24 & $3.95(0.32)$ & $49.4(4.0)$ & $24.0(4.9)$ & $42.8(8.7)$ & $8.8(1.8)$ \\
$2(3.00)$ & 21 & $1.77(0.14)$ & $53.1(4.2)$ & $58.4(4.5)$ & $46.9(3.5)$ & $25.1(1.6)$ \\
$3(4.75)$ & 38 & $1.42(0.11)$ & $67.4(5.2)$ & $64.7(3.5)$ & $40.2(2.8)$ & $24.0(1.6)$ \\
$4(2.12)$ & 22 & $1.50(0.06)$ & $33.4(1.4)$ & $41.6(3.5)$ & $52.9(3.6)$ & $20.3(1.2)$ \\
$5(3.00)$ & 39 & $1.12(0.04)$ & $36.5(1.6)$ & $44.9(0.8)$ & $59.8(2.3)$ & $26.6(0.9)$ \\
$6(3.88)$ & 36 & $1.14(0.07)$ & $44.1(2.9)$ & $53.9(2.8)$ & $59.1(3.5)$ & $29.9(1.4)$ \\
$7(3.00)$ & 27 & $1.16(0.07)$ & $36.3(1.8)$ & $50.3(3.2)$ & $55.0(4.1)$ & $26.5(2.0)$ \\
\hline
\end{tabular}




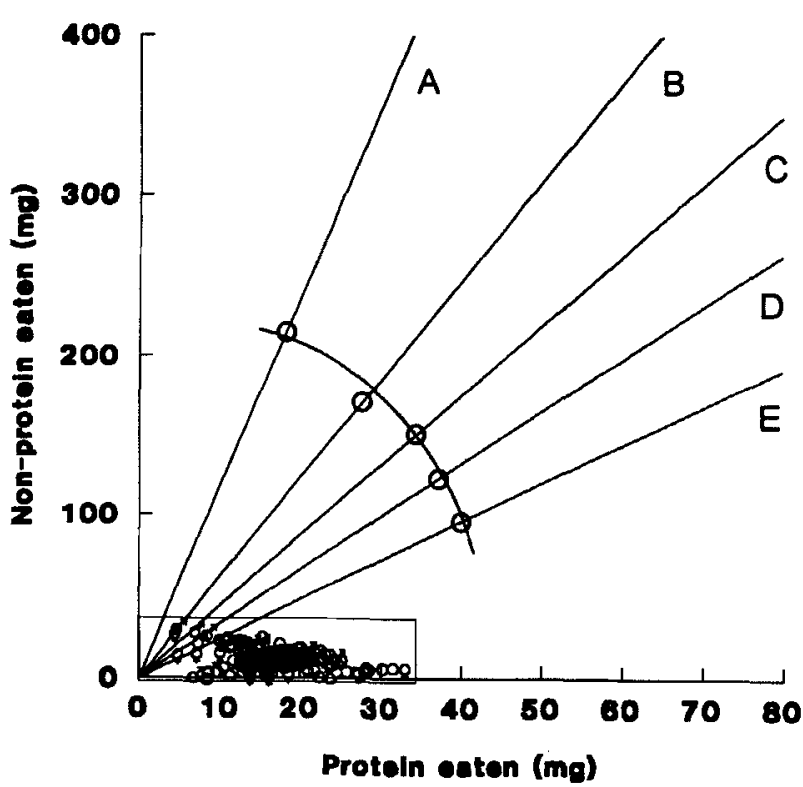

Fig. 4. Intake and growth targets by No Choice larvae as defined by the amounts of protein and non-protein diet components ingested. Lines $\mathrm{A}-\mathrm{E}$ indicate the nutritional 'rails' of $1.25,2.25$, $3.0,3.75$ and $4.75 \% \mathrm{~N}$, respectively. Plotted on each rail is the mean intakc of larvae confined to that rail, and a curve has been drawn through these. The box in the lower left surrounds the estimated growth target of larvae. Open circles designate larvae for which sex was not determined.

promise on their rail or suffer the fitness consequences. The point of best compromise will be dictated largely by the requirements for specific nutrients. Larvae restricted to nutrient rails in the No Choice treatments appeared to adjust their feeding so as to reach the geometrically closest point on the rail to the intake target (Fig. 4). This is evidenced by the curved line that comes closest to the mean intake of larvae on each of the rails. Larvae did not eat a fixed amount of protein common to all the rails (which would be indicated by a vertical line fixed at some amount of protein), nor did they eat a fixed amount of any other diet component (which would be indicated by a horizontal response). Instead, larvae regulated their intake of both protein and some non-protein diet component(s), causing an inward flexing of the mean intake relative to the origin. Note that these data do not define the shape of the curve with high precision; additional points would help confirm the response and differentiate it from the other possible responses discussed by Raubenheimer \& Simpson (1993).

This 'least squares optimization' (McFarland \& Sibly, 1972; Sibly \& McFarland, 1974) was imperfect. Larvae restricted to $3.0 \% \mathrm{~N}$ diet ate $30.5 \mathrm{mg}$ protein and $134.4 \mathrm{mg}$ of non-protein food, amounts virtually identical to the estimated intake target. However, larvae fed $1.25 \% \mathrm{~N}$ food alone ate substantially more food than would be expected if they consumed exactly at the geometrically closest point to the estimated intake target (mean intake lies further from the origin than expected). This 'excess' food consumed could indicate a minimum protein thresh- hold around $19 \mathrm{mg}$ that larvae had to exceed in order to successfully complete the stadium. In order to meet this minimum, larvae needed to eat more of the non-protein diet components than would otherwise be necessary.

Based on previous measures of utilization efficiencies as a function of food protein concentration (Stockhoff, 1992, and unpublished data), the contribution of protein towards growth was estimated. The resulting 'growth target' of larvae lies far below the intake target (inset in Figs 3 and 4). This is necessarily so because of respiration and other 'inefficiencies' in the conversion of consumed food into growth. The estimated amounts of protein and non-protein diet components allocated towards growth are very similar between larvae confined to protein rails and those permitted to choose among different protein concentrations. This indicates the capacity of larvae to 'jump rails' by differentially utilizing consumed nutrients. For larvae in the No Choice treatments, mean protein and non-protein allocated towards growth were $16.8 \mathrm{mg}$ and $11.8 \mathrm{mg}$, respectively. For larvae in the Choice treatments, these amounts were $17.3 \mathrm{mg}$ and $13.5 \mathrm{mg}$, respectively. The nutritional target, defined as the combination of protein and non-protein diet components which provides tissues with the optimal amount and balance of these (Raubenheimer \& Simpson, 1993), will lie up the $y$-axis from the growth target. This is because the non-protein portion of the diets contains carbohydrates, which are the primary source of energy used in respiration.

\section{Discussion}

The experiments described in this paper suggest that gypsy moth larvae may benefit from small-scale, intraplant variation in leaf nutrient composition. This stands in contrast with (but does not necessarily refute) the hypothesis that plant variation has defensive value. There are potentially strong detrimental effects of variation on the growth of gypsy moth larvae (Stockhoff, 1993a), but larvae avoided these by selective feeding. Non-random feeding was evident in that larvae mixed different diet cubes more than they mixed identical diet cubes. Larvae grew as fast or faster on mixed diets as on the component diets alone. Also, when provided with the same mean nitrogen concentration, larvae with a choice grew as fast as or faster than larvae without a choice. A likely contributor to the better growth performance of larvae with a choice than larvae without a choice is that larvae with a choice were able to mix diet cubes so as to match their theoretical intake target more closely. However, larvae unable to mix food items demonstrated a striking capacity to 'jump rails' to reach the same growth target in terms of body composition, if not body size.

Small-scale variation in food quality apparently can have either beneficial or detrimental effects on insects. Larvae that faced variation in food nitrogen concentration without the possibility to choose and set the pattern of changes among items suffered reduced growth and reduced food utilization efficiency (Stockhoff, 1993a). Larvae here 
were provided the same variation in nitrogen concentration but were able to set for themselves the timing of change among items. In either situation, variance clearly may be an important companion measure to the mean when estimating food quality. Average concentrations or ratios of nutrients consumed over extended periods of time probably do not accurately reflect what an individual experiences over short periods of time. As a result, nutrient means or ratios alone may provide misleading estimates of diet quality.

The balance of positive and negative effects of variation will dictate the effectiveness of plant variation as a defence against insect herbivores. Although gypsy moth feed nonrandomly with respect to food nitrogen concentration and benefit from variation in nitrogen, larvae may forage randomly with respect to other traits and suffer negative consequences of variation in these traits. Also, insects may experience variation in multiple traits which may be particularly important in situations where beneficial traits (e.g. nutrients) covary negatively or a detrimental trait covaries positively with a beneficial trait. To assess fully the potential defensive value of variation, other factors must also be assessed, including foraging risks and costs accrued while searching for items. The oft-cited costs associated with foraging may be offset to a significant extent by benefits of variation such as demonstrated here.

Presumably, the functional rule employed by the larvae to regulate nutrient intake reflects the evolutionary pressures placed on the insects. The fact that larvae confined to specific protein concentrations tended to feed as close as possible to the estimated intake target suggests that the insects regulated intake of both protein and non-protein components of the diets. Similar behaviour was reported by Raubenheimer \& Simpson (1993) for the locust, Locusta migratoria L., which regulated intake of both protein and carbohydrate. The regulation by gypsy moth of nonprotein intake is not surprising because many important nutrients were included in the non-protein portion of the diets. What is surprising is that regulation for protein was approximately equivalent to the sum total of regulation for all non-protein diet components. Under natural conditions, regulation of protein intake may be considerably more important to the success of an insect than regulation of any other single nutrient (excepting water, perhaps, which was excluded in this analysis).

Extrapolation to gypsy moth operating under natural conditions is not straightforward, however, because of ecological constraints to compensatory abilities (Slansky \& Scriber, 1985). Although the mechanisms by which insects regulate nutrient intake and 'jump rails' need not be complex (Raubenheimer \& Simpson, 1993), the importance of protein relative to other nutrients will depend critically on the acquisition and processing costs associated with different foods (Martin \& Van't Hof, 1988). Actively foraging larvae, for example, will have higher metabolic costs than experienced by larvae in this study. Both the intake and nutritional targets would be expected to shift to a lower proportion of protein.

Provision with substitutable food items produced be- haviour similar to that of self-selectors provided with strongly complementary items (Waldbauer \& Friedman, 1991). With respect to growth, however, results differed from results with choice between complementary diets obtained from H.zea, another lepidopteran. H.zea larvae with a choice between two cubes that were complementary in protein and carbohydrate (self-selectors) grew better than larvae on component, deficient diets, but performed worse when compared to control larvae provided two cubes of complete diet (Waldbauer et al., 1984). Cohen et al. (1987b) provided evidence that larvae with a choice expended greater energy in travelling between foods, resulting in lower ECD and growth. This was not the case in the present study. Gypsy moth larvae with a choice tended to perform better, not worse than control larvae. One possible explanation for the difference between the two studies may be that gypsy moth larvae performed better because they were already adjusted to the diet treatments by exposure during previous stadias. H.zea larvae were not introduced to the deficient diets until the stadium in which responses were measured. Some form of learning or physiological conditioning may occur during extended periods of exposure such that gypsy moth larvae were better prepared to cope with choices.

Another possible explanation is that nutrient requirements of gypsy moth may have changed during juvenile development, and different diets permitted greater flexibility to meet these changing demands (Simpson \& Simpson, 1990). Such flexibility was hypothesized to explain the much better growth of Supella longipalpa self-selecting between two complementary diets than consuming a single diet containing the self-selected ratio (Cohen et al., 1987a). Time-lapse studies by Cohen et al. (1987b) demonstrated that the selected ratio by $H$.zea changed during a stadium, implying that nutritional requirements also changed. For gypsy moth there is evidence consistent with this hypothesis. Relative preference for protein and lipid changes during juvenile development of gypsy moth (Stockhoff, 1993b), a behaviour that probably reflects changing nutritional demands.

There are several reasons why one might expect consumption and food utilization indices to be unequal between Choice and No Choice treatments, even given the same mean percentage protein. First, as was the case in the High Variance treatments, larvae with a choice will not necessarily feed such that the mean percentage of protein that is consumed is equal to the mean percentage of the protein that is available. If the foods having greater than average concentrations of protein are preferred, then consumption rates would be expected to decline while food utilization efficiencies would increase (Slansky \& Scriber, 1985). Second, larvae with a choice are able to set for themselves the timing of changes from one food type to another. If nutrient requirements change within or across stadia, then this flexibility could lead to greater efficiency in the utilization of individual foods. Third, consumption rate and food utilization efficiency are nonlinear functions of protein concentration, at least for gypsy moth (Stockhoff, 1993a). Specifically, RCR declines rapidly as percentage 
protein in the diet increases, then declines more slowly (e.g. Table 3: note the change in RCR from 3.95 to $1.42 \mathrm{mg} \mathrm{mg}^{-1} \mathrm{~d}^{-1}$. The increase in consumption rate per unit decrease in protein concentration is greater than the decline in consumption rate per unit increase in protein concentration). AD, ECD and ECI tend to increase asymptotically so that each additional unit of protein has diminishing returns in terms of the proportion utilized (the opposite is true from RCR; a unit decrease in protein concentration from $3.0 \% \mathrm{~N}$ has a smaller effect than does a unit increase above $3.0 \% \mathrm{~N}$ ). As a result, one might cxpect that larvae with a choice would have higher RCR but lower AD, ECD and ECI than larvae without a choice, given the same mean percentage protein. The indices reported in Table 3 reflect the sum total of these three factors. Without detailed manipulative experiments it is impossible to determine the relative role of each factor. It is important to note, however, that an absence of differences in indices between Choice and No Choice treatments may be as indicative of compensatory processes as is the presence of differences.

Although unequal allocation of nutrients among plant tissues may fulfill other functions unrelated to herbivory, intraplant variation often is thought to have defensive value by placing herbivores in compromise situations (Schultz, 1983). Because of reduced efficiency in consuming and digesting variable foods and greater search costs (mortality risk, time and metabolic expenditures), an insect foraging among variable leaves will experience a higher risk per unit benefit than one foraging among uniformly high-quality leaves (Schultz, 1983). However, intraplant variation may also benefit insects by providing the opportunity to choose. This benefit will offset the costs associated with variation, perhaps making selective behaviour a more profitable venture than previously thought. In view of the ubiquitous occurrence of variability in the environment, both the positive and negative effects of intraplant variability must be considered when evaluating the net selective pressures placed on herbivores by their hosts and vice versa, and ultimately the evolution of plant-insect systems.

\section{Acknowledgments}

I thank L. Rankin for help in rearing larvae and collecting data. Support was provided by McIntire-Stennis grant MICY -00078 to G. E. Belovsky and J. A. Witter, NSF grant BSR-8904043 to M. M. Martin, and funding by Sigma Xi and the Horace H. Rackham School of Graduate Studies. The work is in partial fulfillment of doctoral degree requirements at the University of Michigan.

\section{References}

Bernays, E.A. \& Bright, K.L. (1991) Dietary mixing in grasshoppers: switching induced by nutritional imbalances in foods. Entomologia Experimentalis et Applicata, 61, 247-253.
Chesson, J. (1983) The estimation and analysis of preference and its relationship to foraging models. Ecology, 64, 1297-1304.

Cohen, R.W., Heydon, S.L., Waldbauer, G.P. \& Friedman, S. (1987a) Nutrient self-selection by the omnivorous cockroach Supella longipalpa. Journal of Insect Physiology, 33, 77-82.

Cohen, R.W., Waldbauer, G.P., Friedman, S. \& Schiff, N.M. (1987b) Nutrient self-selection by Heliothis zea larvae: a timelapse film study. Entomologia Experimentalis et Applicata, 44, 65-73.

Gulmon, S.L. \& Chu, C.C. (1981) The effects of light and nitrogen on photosynthesis, leaf characteristics, and dry matter allocation in the chaparral shrub Diplacus aurantiacus. Oecologia, 49, 207-212.

Hollinger, D.Y. (1989) Canopy organization and foliage synthetic capacity in a broad-leaved evergreen montane forest. Functional Ecology, 3, 53-62.

Lcchowicz, M.J. (1982) The sampling charactcristics of electivity indices. Oecologia, 52, 22-30.

Martin, M.M. \& Van't Hof, H.M. (1988) The cause of reduced growth of Manduca sexta larvac on a low-water diet: increased metabolic processing costs or nutrient limitation? Journal of Insect Physiology, 34, 515-525.

McFarland, D.J. \& Sibly, R. (1972) 'Unitary drives' revisited. Animal Behavior, 20, 548-563.

Odell, T.M., Butt, C.A. \& Bridgeforth, A.W. (1985) Lymantria dispar. Handbook of Insect Rearing, Vol. 2 (ed. by P. Singh and R. F. Moore), pp. 355-367. Elsevier, New York.

Raubenheimer, D. \& Simpson, S.J. (1993) The gcometry of compensatory feeding in the locust. Animal Behaviour, in press.

Schiff, N.M., Waldbauer, G.P. \& Fricdman, S. (1988) Dietary self-selection for vitamins and lipid by larvac of the corn carworm, Heliothis zea. Entomologia Experimentalis et Applicata, 46, 240-256.

Schmidt, D.J. \& Reese, J.C. (1986) Sources of crror in nutritional index studies of insects on artificial diet. Journal of Insect Physiology, 32, 193-198.

Schultz, J.C. (1983) Habitat selection and foraging tactics of caterpillars in heterogeneous trees. Variable Plants and Herbivores in Natural and Managed Systems (ed. by R. F. Denno and M. S. McClurc), pp. 61-90. Academic Press, New York.

Scriber, J.M. (1984) Host-plant suitability. Chemical Ecolngy of Insects (cd. by W. J. Bell and R. T. Cardć), pp. 159-202. Chapman \& Hall, New York.

Scriber, J.M. \& Slansky, F., Jr (1981) The nutritional ecology of immature insects. Annual Review of Entomology, 298. $183-211$.

Sibly, R. \& McFarland, D.J. (1974) A statc-space approach to motivation. Methods of Motivational Control System Analysis (cd. by D. J. McFarland), pp. 213-250. Academic Press, New York.

Simpson, S.J. \& Simpson, C.L. (1990) The mechanisms of compensation by phytophagous insects. Insect-Plant Interactions (ed. by E. A. Bernays), pp. 111-160. CRC Press, Boca Raton.

Slansky, F., Jr \& Scriber, J.M. (1985) Food consumption and utilization. Comprehensive Insect Physiology, Biochemistry and Pharmacology, Vol. 4 (ed. by G. A. Kerkut and L. I. Gilbert), pp. 87-163. Pergamon Press, Oxford.

Stockhoff, B.A. (1992) Dict heterogeneity: effects on gypsy moth feeding bchavior and growth. Doctoral dissertation. University of Michigan, Ann Arbor.

Stockhoff, B.A. (1993a) Dict heterogencity: implications for growth of a generalist herbivorc. the gypsy moth. Ecology, in press.

Stockhoff, B.A. (1993b) Ontogenetic change in dietary selection for protein and lipid by gypsy moth larvac. Journal of Insect 
Physiology, in press.

Tilman, D. (1980) Resources: a graphical-mechanistic approach to competition and predation. American Naturalist, 116, $362-393$

van Loon, J.J.A. (1991) Measuring food utilization in plantfecding insects: toward a metabolic and dynamic approach. Insect-Plant Interactions (ed. by E. A. Bernays), pp. 79-124. CRC Press, Boca Raton.

van Valen, L. (1978) The statistics of variation. Evolutionary Theory, 4, 33-43.

Waldhauer, G.P. (1968) The consumption and utilization of food by insects. Advances in Insect Physiology, 5, 229-288.
Waldbauer, G.P., Cohen, R.W. \& Friedman, S. (1984) Selfselection of an optimal nutrient mix from defined diets by larvae of the corn earworm, Heliothis zea (Boddie). Physiological Zoology, 57, 590-597.

Waldbauer, G.P. \& Friedman, S. (1988) Dietary self-selection by insects. Endocrinological Frontiers in Physiological Insect Ecology (ed. by F. Sehnal, A. Zabza and D. L. Denlinger), pp. 403-422. Wroclaw Technical University Press, Wroclaw. Waldbauer, G.P. \& Friedman, S. (1991) Self-selection of optimal diets by insects. Annual Review of Entomology, 36, 43-63.

Accepted 20 May 1993 